\title{
CONDITIONALLY INDEPENDENT INCREMENT POINT PROCESSES
}

\author{
RICARDO VÉLEZ IBARROLA* AND \\ TOMÁS PRIETO-RUMEAU, ${ }^{* * *}$ Universidad Nacional de Educación a Distancia
}

\begin{abstract}
In this paper we introduce conditionally independent increment point processes, that is, processes that are conditionally independent inside and outside a bounded set $A$ given $N(A)$, the number of points in $A$. We show that these point processes can be characterized by means of the avoidance function of a multinomial 'support process', the solution of a suitably defined linear system of equations, and, finally, the infinitesimal matrix of a continuous-time Markov chain.
\end{abstract}

Keywords: Markov and multinomial point processes; continuous-time Markov chain

2010 Mathematics Subject Classification: Primary 60G55; 60J27

\section{Introduction}

In the theory of point processes, the Poisson processes are probably the most widely studied. Their main feature is the so-called complete independence property; that is, given bounded and disjoint measurable sets $A_{1}, \ldots, A_{k}$, the random variables $N\left(A_{1}\right), \ldots, N\left(A_{k}\right)$ are independent, where $N\left(A_{i}\right)$ for $1 \leq i \leq k$ stands for the number of points in the set $A_{i}$.

For practical purposes, however, the complete independence property can be too restrictive. Several generalizations aimed towards weakening the independence property have been proposed in the literature. These include the Poisson cluster processes, which are derived from a 'parent' Poisson process, Neyman-Scott processes, and bombing models, to name just a few; see, e.g. [4, Chapter 4], [7, Section 5.5], and [9, Section 1.6]. These generalizations are, however, mainly restricted to models for which the underlying Poisson process has finite intensity measure, thus putting aside many interesting cases with infinite intensity measure.

In this paper we replace the complete independence assumption made for Poisson processes with a weaker conditional independence requirement. Roughly speaking, the conditional independence assumption states that, given a bounded measurable set $A$, the process inside the set $A$ is conditionally independent of the process outside the set $A$ given the random variable $N(A)$. This assumption has a Markovian flavor; indeed, it looks very similar to the definition of a Markov process on the real line, in the sense that the future is conditionally independent of the past given the present. Thus, we will also call such processes Markovian point processes.

At this point, it is worth noting that these processes should not be confused with the Markov point processes of [9], which are defined in a different way. More precisely, in [9] the process inside $A$ is conditionally independent of the process outside $A$ given the process on a set

Received 5 October 2009; revision received 19 May 2010.

* Postal address: Departamento de Estadística, Universidad Nacional de Educación a Distancia (UNED), Calle Senda del Rey 9, 28040 Madrid, Spain.

** Email address: tprieto@ccia.uned.es 
outside $A$ but 'close enough' to $A$. In spite of this, we must admit that the term Markovian is rather appealing for processes with the conditional independence property stated above, and, hence, they will be referred to as Markovian point processes as well, without any risk of misunderstanding.

In this paper we will consider a Markovian point process $\mathcal{N}$ on a complete and separable metric space, defined on an arbitrary probability space. The Markov property implies that the finite-dimensional distributions of $\mathcal{N}$ are determined by

(i) the marginal distribution of $N(A)$; and

(ii) the conditional distribution of $N(B)$ given $N(A)$,

for arbitrary bounded measurable sets $B \subseteq A$. Hence, our main efforts are aimed towards the characterization of the marginal distributions in (i) and the conditional distributions in (ii).

The rest of the paper is organized as follows. In Section 2 we give a precise definition of a Markovian point process, and we also introduce some useful concepts, such as regularity or homogeneity of a Markovian point process. In Section 3 we analyze the Markovian point processes which, in addition, are simple. Under some additional hypotheses, we prove that they are multinomial point processes (which are somehow similar to mixed Poisson point processes). Section 4 is devoted to the study of general Markovian point processes. We give a complete characterization of their conditional distributions. Also, we show that Markovian point processes are marked multinomial point processes and, finally, we give sufficient conditions for constructing the marginal distributions of a Markovian point process. In Section 5 we give an overview of the results in this paper, and, in particular, we show how to construct (or characterize) an arbitrary Markovian point process from its basic elements. We also mention some interesting open issues.

\section{Basic definitions}

We consider an arbitrary complete and separable metric space $(E, d)$ endowed with its Borel $\sigma$-algebra $\mathcal{E}$. The ring of bounded sets in $\mathcal{E}$ will be denoted by $\mathcal{B}$. Given $A \in \mathcal{E}$, by $\mathcal{E}_{A}$ we will denote the restriction of the $\sigma$-algebra $\&$ to $A$, that is,

$$
\varepsilon_{A}=\{A \cap B \mid B \in \mathcal{E}\} .
$$

Let $\mathcal{N} \equiv\{N(A)\}_{A \in \mathcal{E}}$ be a point process defined on a probability space $(\Omega, \mathcal{F}, \mathrm{P})$, whose realizations are integer-valued, boundedly finite measures on $(E, \mathcal{E})$; see $[3$, Section 7.1]. Given $A \in \mathcal{E}$, by $\mathcal{F}_{A}$ we will denote the sub- $\sigma$-field of $\mathcal{F}$ generated by the random variables $\{N(B): B \in \mathcal{E}, B \subseteq A\}$.

Let us introduce some notation. Given a boundedly finite measure $\mu$ on $(E, \mathcal{E})$, we will denote by $R_{\mu}$ the range of $\mu$ over the family of bounded sets, that is, $R_{\mu}=\{\mu(A) \mid A \in \mathscr{B}\}$. Given $a, b \in R_{\mu}$, we will write $a \prec b$ if there exist $A \subseteq B \in \mathscr{B}$ such that $\mu(A)=a$ and $\mu(B)=b$.

\subsection{Markovian point processes}

By a Markovian point process $\mathcal{N}$ we mean that, when $N(A)=i$, the allocation of the $i$ points inside $A$ is independent of the behavior of the process outside $A$. It should be noted that this definition is different from the concept of a Markov point process considered in [9], where the conditional independence is assumed to hold given the process in some 'neighborhood' of $A$; see, e.g. [9, Theorem 2.1]. Without risk of confusion, we define Markovian point processes to be those satisfying the following definition. 
Definition 2.1. A point process $\mathcal{N}$ will be called Markovian if, for all $A \in \mathscr{B}$, the $\sigma$-fields $\mathcal{F}_{A}$ and $\mathcal{F}_{A^{\mathrm{c}}}$ are conditionally independent given $N(A)$.

It is easily seen that the finite-dimensional distributions of a Markovian point process $\mathcal{N}$ are determined by the following elements:

(a) the family of probability row vectors $p_{A}$, with $p_{A}(i)=\mathrm{P}\{N(A)=i\}$ for $i=0,1, \ldots$ and $A \in \mathcal{B}$;

(b) the family of lower triangular stochastic matrices $P_{A, B}$, where

$$
P_{A, B}(i, j)=\mathrm{P}\{N(B)=j \mid N(A)=i\}
$$

for $0 \leq j \leq i$ and $A, B \in \mathscr{B}$ with $B \subseteq A$.

Indeed, if $B_{1}, \ldots, B_{r} \in \mathscr{B}$ are disjoint sets then $\mathrm{P}\left\{N\left(B_{1}\right)=i_{1}, \ldots, N\left(B_{r}\right)=i_{r}\right\}$ equals

$$
p_{A}(i) P_{A, B_{1}}\left(i, i_{1}\right) P_{A-B_{1}, B_{2}}\left(i_{2}+\cdots+i_{r}, i_{2}\right) \cdots P_{B_{r-1} \cup B_{r}, B_{r-1}}\left(i_{r-1}+i_{r}, i_{r-1}\right),
$$

where $A=B_{1} \cup \cdots \cup B_{r}$ and $i=i_{1}+\cdots+i_{r}$.

From Theorem 7.1.XI of [3], it is straightforward to derive necessary and sufficient conditions ensuring that a family of probability vectors $p_{A}$ and lower triangular stochastic matrices $P_{A, B}$ determines the distribution of a Markovian point process.

Proposition 2.1. A family of probability row vectors $p_{A}$ (with $A \in \mathscr{B}$ ) and lower triangular stochastic matrices $P_{A, B}$ (with $B \subseteq A$ in $\mathscr{B}$ ) determines the finite-dimensional distributions of a Markovian point process if and only if the following conditions are satisfied:

(i) $P_{A, A-B}(i, j)=P_{A, B}(i, i-j)$ for all $0 \leq j \leq i$;

(ii) $P_{A, B_{1}}\left(i, j_{1}\right) P_{A-B_{1}, B_{2}}\left(i-j_{1}, j_{2}\right)=P_{A, B_{2}}\left(i, j_{2}\right) P_{A-B_{2}, B_{1}}\left(i-j_{2}, j_{1}\right)$ for $i \geq j_{1}+j_{2}$, whenever $B_{1}$ and $B_{2}$ are disjoint bounded measurable subsets of $A$;

(iii) $P_{A, B} P_{B, C}=P_{A, C}$ if $C \subseteq B \subseteq A$, where $A, B, C \in \mathscr{B}$;

(iv) $p_{B}=p_{A} P_{A, B}$;

(v) $p_{B_{n}}(0) \rightarrow 1$ when $B_{n} \downarrow \varnothing$.

Observe that Proposition 2.1(i) and (ii) ensure that (2.1) does not depend on the order of the partition of $A$, while (i) and (iv) allow us to derive the marginal distribution of $\left(N\left(B_{2}\right), \ldots\right.$, $N\left(B_{r}\right)$ ) by summing (2.1) over $i_{1}$. The multiplicative property in (iii) is an obvious compatibility assumption, similar to the Chapman-Kolmogorov equation, yielding, together with (i), the Equation (7.1.9) of [3].

Since $p_{B_{n}}=p_{A} P_{A, B_{n}}$, if $B_{n} \subseteq A$, Proposition 2.1(v) may be reformulated as $P_{A, B_{n}} \rightarrow P_{0}$ (componentwise), where $P_{0}$ is the stochastic matrix whose first column terms are 1. By (i), this is equivalent to $P_{A, B_{n}} \rightarrow I$ (componentwise) if $B_{n} \uparrow A$, where $I$ is the identity matrix. Condition (iv) implies that knowledge of the distribution $p_{A}$ for large sets (for instance, for a sequence $\left\{E_{n}\right\}$ of bounded sets increasing to $E$ ) suffices to determine the distribution $p_{B}$ for smaller sets. 


\subsection{Regular point processes}

Suppose that $\mu$ is a measure on a topological space $\mathcal{X}$ endowed with its Borel $\sigma$-algebra. We say that $\mu$ is supported on $\mathcal{X}$ if $\mu(A)>0$ for any nonempty open subset of $\mathcal{X}$.

Definition 2.2. A point process $\mathcal{N}$ will be called regular if

$$
\mathrm{P}\{N(B)=1, N(A-B)=0\}>0
$$

for any bounded nonempty open sets $A$ and $B$ such that $B \subseteq A$.

The regularity hypothesis means that there is a positive probability that there is a unique point in any bounded nonempty open set, and, further, that the probability measure on $A$ defined by $B \mapsto \mathrm{P}\{N(B)=1 \mid N(A)=1\}$ is supported on $A$. As an illustration, a Poisson process with intensity measure supported on $E$ is regular.

Theorem 2.1. If $\mathcal{N}$ is a regular Markovian point process then there exists a boundedly finite measure $m$ on $(E, \mathcal{E})$ supported on $E$ such that

$$
\mathrm{P}\{N(B)=1 \mid N(A)=1\}=\frac{m(B)}{m(A)}
$$

for any $A \in \mathscr{B}$ with $m(A)>0$ and any measurable $B \subseteq A$. Moreover, $m$ is unique up to a multiplicative constant.

Proof. If $\mathcal{N}$ is a regular Markovian point process and $A$ is a nonempty open set, we can consider

$$
m_{A}(B)=\mathrm{P}\{N(B)=1 \mid N(A)=1\} \text { for } B \in \mathcal{E}_{A},
$$

which is a probability measure on the Borel subsets of $A$. Furthermore, if $B$ is a nonempty open subset of $A$ and $C \in \mathcal{E}_{B}$, we have

$$
\begin{aligned}
& \mathrm{P}\{N(C)=1 \mid N(A)=1\} \\
& \quad=\mathrm{P}\{N(B)=1 \mid N(A)=1\} \mathrm{P}\{N(C)=1 \mid N(B)=1, N(A-B)=0\} \\
& \quad=\mathrm{P}\{N(B)=1 \mid N(A)=1\} \mathrm{P}\{N(C)=1 \mid N(B)=1\},
\end{aligned}
$$

and, therefore,

$$
m_{A}(C)=m_{A}(B) m_{B}(C) .
$$

Fix a point $a \in E$, and consider $E_{n}=\{x \in E \mid d(x, a)<n\}$, which is a sequence of bounded open sets increasing to $E$. Since the regularity hypothesis implies that $m_{E_{n}}\left(E_{1}\right)>0$, the finite measure

$$
m_{n}(A)=\frac{m_{E_{n}}(A)}{m_{E_{n}}\left(E_{1}\right)} \quad \text { for } A \in \mathcal{E}_{E_{n}}
$$

is such that $m_{n}$ agrees with $m_{n-1}$ on $E_{n-1}$ because, when $A \in \mathcal{E}_{E_{n-1}}$, it is

$$
m_{n}(A)=\frac{m_{E_{n}}\left(E_{n-1}\right) m_{E_{n-1}}(A)}{m_{E_{n}}\left(E_{n-1}\right) m_{E_{n-1}}\left(E_{1}\right)}=m_{n-1}(A) .
$$

Consequently, we obtain a unique measure defined, for any set $A \in \mathscr{B}$, as

$$
m(A)=m_{n}(A) \text { for all } n \text { such that } A \subset E_{n},
$$


and $m$ can be extended to unbounded Borel sets. Obviously, $m$ is boundedly finite since $m\left(E_{n}\right)=m_{E_{n}}\left(E_{1}\right)^{-1}$, and $m(A)>0$ for any nonempty open set $A$. Furthermore, if $B \subseteq A \subset$ $E_{n}$ and $m(A)>0$, it is

$$
\frac{m(B)}{m(A)}=\frac{m_{E_{n}}(B)}{m_{E_{n}}(A)}=\frac{m_{E_{n}}(A) m_{A}(B)}{m_{E_{n}}(A)}=m_{A}(B)=\mathrm{P}\{N(B)=1 \mid N(A)=1\} .
$$

Finally, assume that $m^{\prime}$ is another measure for which the result holds. In this case, if $A \in \mathscr{B}$ and $A \subseteq E_{n}$, we have

$$
m^{\prime}(A)=\frac{m^{\prime}\left(E_{n}\right)}{m\left(E_{n}\right)} m(A)=c_{n} m(A) ;
$$

hence, $c_{n}$ does not depend on $n$, and $m^{\prime}(A)=c m(A)$. This completes the proof.

Theorem 2.1 makes use of the regularity assumption only to construct a sequence of bounded open sets $E_{n} \uparrow E$ such that

$$
\mathrm{P}\left\{N\left(E_{n}\right)=1, N\left(E_{n}-E_{1}\right)=0\right\}>0 \text { for all } n .
$$

By assuming the existence of such a sequence, we could drop the hypothesis that $E$ is a complete and separable metric space, and still prove this result in the context of an abstract measurable space $(E, \mathcal{E})$, along the line proposed in [8].

\subsection{Symmetric point processes}

We say that a point process $\mathcal{N}$ is symmetrically distributed with respect to a boundedly finite measure $\mu$ on $(E, \mathcal{E})$ if (cf. [5, p. 73])

$$
\left[N\left(B_{1}\right), \ldots, N\left(B_{r}\right)\right] \stackrel{\mathrm{D}}{=}\left[N\left(B_{1}^{\prime}\right), \ldots, N\left(B_{r}^{\prime}\right)\right]
$$

whenever $\left\{B_{l}\right\}_{1 \leq l \leq r}$ and $\left\{B_{l}^{\prime}\right\}_{1 \leq l \leq r}$ are families of disjoint sets in $\mathscr{B}$ such that $\mu\left(B_{l}\right)=\mu\left(B_{l}^{\prime}\right)$ for each $l=1, \ldots, r$.

Obviously, for a Markovian point process $\mathcal{N}$ symmetrically distributed with respect to $\mu$,

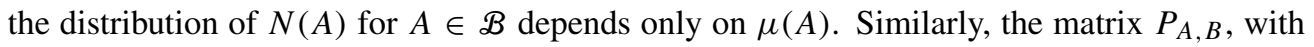
$B \subseteq A \in \mathscr{B}$, depends only on $\mu(A)$ and $\mu(B)$. Therefore, in this case, we will write

$$
p(a)=p_{A} \quad \text { and } \quad P_{a, b}=P_{A, B},
$$

where $a=\mu(A)$ and $b=\mu(B)$ for $A, B \in \mathscr{B}$. For a symmetric Markovian point process which, in addition, is regular, we obtain the next result.

Proposition 2.2. If $\mathcal{N}$ is a regular Markovian point process symmetrically distributed with respect to some boundedly finite, nonpurely atomic measure $\mu$, then $\mu$ is the measure $m$ given in Theorem 2.1.

Proof. For disjoint sets $B, B^{\prime} \in \mathcal{B}$ with $\mu(B)=\mu\left(B^{\prime}\right)$, given any $A \in \mathscr{B}$ such that $A \supseteq B \cup B^{\prime}$, since $P_{A, B}$ depends only on the distribution of $[N(B), N(A-B)]$, we have $P_{A, B}=P_{A, B^{\prime}}$

Moreover, $\mathcal{N}$ being regular, Theorem 2.1 holds, and so $m(B)=m\left(B^{\prime}\right)$. Hence, $m$ is a function of $\mu: m(B)=f(\mu(B))$, where $f$ must be increasing, linear, and such that $f(0)=0$. But, $\mu$ being nonpurely atomic, its range necessarily contains some interval $[0, \delta]$. Therefore, $m(B)=c \mu(B)$, and the stated result follows. 


\subsection{Homogeneous symmetric Markovian point processes}

For a Markovian point process $\mathcal{N}$ symmetric with respect to a measure $\mu$, it seems sensible to assume that

$$
P_{A, B}(i, j)=\mathrm{P}\{N(B)=j \mid N(A)=i\}
$$

should be the same for any $B \subseteq A$ in $\mathscr{B}$ whose relative size (with respect to the measure $\mu$ ) is the same. In our next definition, we make precise this idea.

Definition 2.3. A Markovian point process $\mathcal{N}$ symmetric with respect to the boundedly finite measure $\mu$ will be said to be homogeneous with respect to $\mu$ if $P_{c a, c b}=P_{a, b}$ for every $a, b \in R_{\mu}$ and $c>0$ such that $b \prec a$ and $c b \prec c a$.

It then follows that $P_{a, b}$ is the same matrix $P(b / a)$ for all $b \prec a$. As a consequence, the distribution of a homogeneous Markovian point process with respect to a nonpurely atomic measure $\mu$ is determined by

(a) a family of probability (row) vectors $p(a)$ for $a \in R_{\mu}$;

(b) a family of (lower) triangular stochastic matrices $P(x)$ for $0 \leq x \leq 1$.

Note that the fact that $\mu$ is nonpurely atomic is needed to ensure that, for every $x \in[0,1]$, there exist $b \prec a$ such that $b / a=x$. The result of Proposition 2.1 is easily adapted to the case of a homogeneous Markovian point process.

Proposition 2.3. Suppose that a boundedly finite, nonpurely atomic measure $\mu$ on $(E, \mathcal{E})$ is given. A family of probability row vectors $p(a)$ (with $a \in R_{\mu}$ ) and lower triangular stochastic matrices $P(x)$ (with $0 \leq x \leq 1)$ determines the finite-dimensional distributions of a Markovian point process $\mathcal{N}$, symmetric and homogeneous with respect to $\mu$, if and only if the following conditions are satisfied:

(i) $P_{i, j}(1-x)=P_{i, i-j}(x)$ for all $j \leq i$ and $x \in[0,1]$;

(ii) for all $i \geq j_{1}+j_{2}$ and $x_{1}+x_{2} \leq 1$,

$$
P_{i, j_{1}}\left(x_{1}\right) P_{i-j_{1}, j_{2}}\left(\frac{x_{2}}{1-x_{1}}\right)=P_{i, j_{2}}\left(x_{2}\right) P_{i-j_{2}, j_{1}}\left(\frac{x_{1}}{1-x_{2}}\right) ;
$$

(iii) $P(x) P(y)=P(x y)$ for all $x, y \in[0,1]$;

(iv) $P(x) \rightarrow I$ componentwise as $x \uparrow 1$ (or $P(x) \rightarrow P_{0}$ componentwise as $\left.x \downarrow 0\right)$;

(v) $p(a x)=p(a) P(x)$ for all $a \in R_{\mu}$ and $x \in[0,1]$ such that ax $\prec a$.

This leads to the next definition.

Definition 2.4. A family of lower triangular stochastic matrices $\{P(x)\}_{x \in[0,1]}$ satisfying the conditions (i)-(iv) of Proposition 2.3 will be called an allocation function.

It follows from Proposition 2.3(iii) and (iv) that $P(x)$ is a continuous function of $x \in[0,1]$. Moreover, by making the change of variable $x=\mathrm{e}^{-t}$ for $t \geq 0$, and thus defining $Q(t)=$ $P\left(\mathrm{e}^{-t}\right)$, it follows that $Q(t) Q(s)=Q(s+t)$ for any $s, t \in[0, \infty)$. Therefore, the $\{Q(t)\}_{t \geq 0}$ are the transition matrices of a homogeneous continuous-time Markov chain, which decreases to the absorbing state 0 . As is well known (see, e.g. [2, Chapter 2]), the infinitesimal matrix $Q^{\prime}(0)$, as well as $P^{\prime}(1)$, exists and completely determines $Q(t)$ for every $t \geq 0$, or $P(x)$ for every $x \in[0,1]$. 
Proposition 2.3(i) and (ii), however, impose further restrictions on $P(x)$, so that $Q^{\prime}(0)$ cannot be chosen as an arbitrary $Q$-matrix for a Markov chain decreasing to 0 . The terms of the matrix $Q^{\prime}(0)$ will be denoted by $q_{i, j}$ for $i, j \geq 0$; we define also $q_{i}=-q_{i, i} \geq 0$ for $i \geq 0$.

Remark 2.1. Proposition 2.3(i) and (iii) imply that $P_{i, i}(x)=x^{q_{i}}$ with $q_{i}>0$, and then $P_{i, 0}(x)=(1-x)^{q_{i}}$. In particular, since $P_{1,0}(x)+P_{1,1}(x)=1$ for $x \in[0,1]$, it follows that the first two rows of $P(x)$ are necessarily

$$
\left[\begin{array}{cccc}
1 & 0 & 0 & \cdots \\
1-x & x & 0 & \cdots
\end{array}\right]
$$

As a direct consequence of Remark 2.1, given $B \subseteq A \in \mathcal{B}$, it is

$$
\mathrm{P}\{N(B)=1 \mid N(A)=1\}=\frac{\mu(B)}{\mu(A)}
$$

and

$$
\mathrm{P}\{N(B)=1, N(A-B)=0\}=\frac{p_{1}(\mu(A)) \mu(B)}{\mu(A)},
$$

which yield the following result.

Corollary 2.1. A Markovian point process, homogeneous with respect to a nonpurely atomic measure $\mu$, is regular if and only if $p_{1}(a)>0$ for all $a>0$ in $R_{\mu}$, and, in addition, $\mu$ is supported on $E$.

Remark 2.2 below provides an interpretation of the terms of $Q^{\prime}(0)$ associated with a homogeneous Markovian point process. Let $\mathcal{N}$ be a Markovian point process, homogeneous with respect to $\mu$. Given a set $A \in \mathcal{B}$, suppose that there exists a family of measurable subsets of $A$, $\left\{A_{x}\right\}_{x \in[0,1]}$, decreasing as $x$ decreases from 1 to 0 , and such that $\mu\left(A_{x}\right)=x \mu(A)$ for $0 \leq x \leq 1$. (For instance, if $E=\mathbb{R}^{d}, A$ is a convex set, and $\mu$ is absolutely continuous with respect to the Lebesgue measure, we can fix a point $z_{0} \in A$ and then consider $A_{r}^{\prime}=\left\{z_{0}+r\left(z-z_{0}\right) \mid z \in A\right\}$ for each $r \in[0,1]$. Since $\mu\left(A_{r}^{\prime}\right)=\alpha(r) \mu(A)$, where $\alpha$ is a continuous function of $r$ decreasing from 1 to 0 as $r$ decreases, we can take $A_{x}=A_{\alpha^{-1}(x)}^{\prime}$ as an adequate reparametrization of the homothetic sets $A_{r}^{\prime}$.) Now consider the stochastic process $\left\{N\left(A_{x}\right)\right\}_{1 \geq x \geq 0}$, where $x$ decreases from 1 to 0 . The so-defined Markov process is a reparametrization (by means of $x=\mathrm{e}^{-t}$ ) of a continuous-time Markov chain with transition matrices $Q(t)$ for $t \geq 0$, and with infinitesimal matrix $Q^{\prime}(0)$. Therefore, we can state our next remark.

Remark 2.2. Let $\mathcal{N}$ be a homogeneous Markovian point process with respect to a nonpurely atomic measure, with corresponding infinitesimal matrix $Q^{\prime}(0)$. The process $\left\{N\left(A_{x}\right)\right\}_{1 \geq x \geq 0}$ defined above satisfies the following conditions.

(i) If $N(A)=i$ then the random variable $\xi_{i}=\max \left\{x \in[0,1] \mid N\left(A_{x}\right)<i\right\}$ is distributed with density $q_{i} u^{q_{i}-1}$ on $[0,1]$ (indeed, $-\log \xi_{i}$ is exponentially distributed with parameter $\left.q_{i}\right)$.

(ii) $\mathrm{P}\left\{N\left(A_{\xi_{i}}\right)=j \mid N(A)=i\right\}=q_{i, j} / q_{i}$.

The easiest example of an allocation function is

$$
P_{i, j}(x)=\left(\begin{array}{c}
i \\
j
\end{array}\right) x^{j}(1-x)^{i-j} \quad \text { for } 0 \leq j \leq i \text { and } 0 \leq x \leq 1,
$$


associated to the infinitesimal matrix $Q^{\prime}(0)$ with terms

$$
-q_{i, i}=q_{i}=i \quad \text { and } \quad q_{i, i-1}=i \quad \text { for } i \geq 1, \quad \text { and } \quad q_{i, j}=0 \quad \text { otherwise. }
$$

In this case, conditional on $N(A)=i$, the $i$ points are independently allocated inside $A$ with distribution $\mu(\mathrm{d} x) / \mu(A)$.

Our plan in Sections 3 and 4 below is as follows. We now know that a homogeneous Markovian point process is characterized by its allocation function $P(x)$ and the family $\{p(a)\}$ of its marginal distributions. In Section 3 we propose several sufficient conditions to ensure that a Markovian point process is multinomial (hence, its allocation function is given by (2.2)), and also we propose a characterization of its marginal distributions. In Section 4 we analyze the case of nonmultinomial homogeneous Markovian point process, and we give a characterization of the infinitesimal matrix $Q^{\prime}(0)$ of an allocation function.

\section{Markovian and multinomial point processes}

Let $\mathcal{N}$ be a point process on the complete and separable metric space $E$.

Definition 3.1. We say that the point process $\mathcal{N}$ is multinomial, directed by the boundedly finite measure $\mu$ on $(E, \mathcal{E})$, if, for all $A \in \mathcal{B}$ and any measurable partition $B_{1}, \ldots, B_{k}$ of $A$, we have

$$
\begin{aligned}
& \mathrm{P}\left\{N\left(B_{1}\right)=n_{1}, \ldots, N\left(B_{k}\right)=n_{k} \mid N(A)=n\right\} \\
& \quad=\left(\begin{array}{c}
n \\
n_{1}, \ldots, n_{k}
\end{array}\right)\left(\frac{\mu\left(B_{1}\right)}{\mu(A)}\right)^{n_{1}} \cdots\left(\frac{\mu\left(B_{k}\right)}{\mu(A)}\right)^{n_{k}}
\end{aligned}
$$

for all $n \geq 1$ and $n_{1}, \ldots, n_{k}$ with $n_{1}+\cdots+n_{k}=n$.

A direct calculation shows that if $\mathcal{N}$ is multinomial, directed by $\mu$, then $\mathcal{N}$ is symmetric with respect to $\mu$ and, moreover, $\mathcal{N}$ is Markovian and homogeneous. Also, it is easily seen that if $\mathcal{N}$ is a multinomial point process directed by a diffuse measure $\mu$ then $\mathcal{N}$ is simple.

Our next theorem explores some properties of multinomial point processes, but first we introduce some more notation. Recall that $R_{\mu}$ denotes the range of the measure $\mu$ over the family of bounded measurable sets $\mathscr{B}$. Let $\tilde{R}_{\mu}$ be the smallest interval containing $R_{\mu}$, and note that $\tilde{R}_{\mu}$ can be either $[0, \mu(E))$ or $[0, \mu(E)]$ when $\mu(E)<\infty$, or $\tilde{R}_{\mu}=[0, \infty)$ when $\mu(E)=\infty$.

Theorem 3.1. Let $\mathcal{N}$ be a multinomial point process directed by a boundedly finite measure $\mu$ on $(E, \mathcal{E})$. There exists a continuous and nonnegative function $\psi$ on $\tilde{R}_{\mu}$, with $\psi(0)=1$, such that, for any disjoint sets $A_{1}, \ldots, A_{j}$ in $\mathscr{B}$ and real numbers $0 \leq z_{1}, \ldots, z_{j} \leq 1$,

$$
\mathrm{E}\left[z_{1}^{N\left(A_{1}\right)} \cdots z_{j}^{N\left(A_{j}\right)}\right]=\psi\left(\mu\left(A_{1}\right)\left(1-z_{1}\right)+\cdots+\mu\left(A_{j}\right)\left(1-z_{j}\right)\right) .
$$

Moreover, the function $\psi$ has derivatives of any order $k$ on $\tilde{R}_{\mu}-\{0\}$, which satisfy

$$
(-1)^{k} \psi^{(k)}(x) \geq 0 \text { and } \sum_{k=0}^{\infty} \frac{1}{k !} \psi^{(k)}(x)(-x)^{k}=1 \quad \text { for all } x \in \tilde{R}_{\mu}-\{0\} .
$$

Proof. Suppose that $x \in \tilde{R}_{\mu}$, and fix $a \in R_{\mu}$ such that $x \leq a$. Let $G(a, z)$ for $0 \leq z \leq 1$ be the generating function of $N(A)$ for a set $A \in \mathscr{B}$ with $\mu(A)=a$; that is, $G(a, z)=$ $\sum_{n=0}^{\infty} p_{n}(a) z^{n}$. We define $\psi(x)=G(a, 1-x / a)$. 
Our first task is to show that the function $\psi$ is well defined. To this end, let $b \in R_{\mu}$ be such that $0 \leq x \leq b$, and let us prove that

$$
G\left(a, 1-\frac{x}{a}\right)=G\left(b, 1-\frac{x}{b}\right) .
$$

Suppose that $a \leq b$. If $a \prec b$, it follows from the fact that $\mathcal{N}$ is multinomial that the marginal distributions $p(a)$ and $p(b)$ are related via

$$
p_{k}(a)=\sum_{n=k}^{\infty} p_{n}(b)\left(\begin{array}{l}
n \\
k
\end{array}\right)\left(\frac{a}{b}\right)^{k}\left(1-\frac{a}{b}\right)^{n-k} \text { for } k \geq 0 .
$$

Therefore, $G(a, z)=G(b, 1-a(1-z) / b)$ for $0 \leq z \leq 1$, and (3.3) follows. Suppose now that $a \nprec b$. Then there exists $c \in R_{\mu}$ with $a \prec c$ and $b \prec c$, and (3.3) also follows by the same arguments.

The continuity of $\psi$ on $\tilde{R}_{\mu}$, the fact that $\psi(0)=1$, and the properties of the derivatives of $\psi$ in (3.2) are now easily verified. Finally, the formula for the generating function of $\left[N\left(A_{1}\right), \ldots, N\left(A_{j}\right)\right]$ in $(3.1)$ is derived from the multinomial property of $\mathcal{N}$ and the definition of $\psi$.

We conclude with an important remark.

Remark 3.1. Given a bounded measurable set $A \in \mathscr{B}$ with $\mu(A)=a$, its generating function $G(a, z)$ verifies

$$
\mathrm{E}\left[z^{N(A)}\right]=G(a, z)=\psi(a(1-z)) \quad \text { for } 0 \leq z \leq 1 .
$$

Thus, $\mathrm{P}\{N(A)=0\}=G(a, 0)=\psi(a)$, and we conclude that $\psi$, when restricted to $R_{\mu}$, is the avoidance function of the multinomial point process $\mathcal{N}$.

\subsection{Marginal distributions of a multinomial point process}

Let $\mathcal{N}$ be a multinomial point process directed by the boundedly finite measure $\mu$. We consider two cases, depending on whether $\mu(E)=\infty$ or $\mu(E)<\infty$.

Case 1: $\mu(E)=\infty$. Suppose that $A$ is a bounded measurable set in $\mathcal{B}$. Given $u \geq 0$ and letting $z=\mathrm{e}^{-u / \mu(A)}$ in (3.4), we find that the Laplace transform of $N\left(A_{n}\right) / \mu\left(A_{n}\right)$ is

$$
\mathrm{E}\left[\mathrm{e}^{-u N(A) / \mu(A)}\right]=\psi\left(\mu(A)\left(1-\mathrm{e}^{-u / \mu(A)}\right)\right) .
$$

If $A \uparrow E$ (and, hence, $\mu(A) \uparrow \infty$ ) then $\mathrm{E}\left[\mathrm{e}^{-u N(A) / \mu(A)}\right] \rightarrow \psi(u)$, and it follows from the continuity theorem for Laplace transforms that $\psi$ is the Laplace transform of a nonnegative random variable, say $\Lambda$, and, moreover, that

$$
\frac{N(A)}{\mu(A)} \stackrel{\mathrm{D}}{\rightarrow} \Lambda \quad \text { as } A \uparrow E .
$$

In the case when $\psi^{\prime}(0)$ exists and is finite, $N(A)$ has finite expectation equal to $-\psi^{\prime}(0) \mu(A)$, and the above convergence is also almost sure. Indeed, this is derived, taking into account the symmetry property, from standard exchangeability results [1, p. 227] applied to the sequence $\left\{N\left(A_{k}\right)\right\}_{k \geq 1}$, where the $\left\{A_{k}\right\}_{k \geq 1}$ are disjoint sets in $\mathcal{B}$ with $\mu\left(A_{1}\right)=\mu\left(A_{2}\right)=\cdots$.

Conversely, suppose that $\psi$ is the Laplace transform of a nonnegative random variable $\Lambda$. We say that $\mathcal{N}^{\prime}$ is a mixed Poisson process (see, e.g. [3, Example 7.4(b)]) directed by the 
boundedly finite measure $\mu$ with mixing random variable $\Lambda$ if, conditional on $\Lambda=\lambda, \mathcal{N}^{\prime}$ is a Poisson process with intensity measure $\lambda \mu$. It is easy to check that, in this case, the generating functions of $\mathcal{N}^{\prime}$ are precisely as in (3.1). Therefore, $\mathcal{N}$ is a multinomial point process directed by $\mu$ if and only if it is a mixed Poisson process directed by $\mu$. Moreover, the avoidance function of $\mathcal{N}$ and the Laplace transform of the mixing random variable coincide.

Case 2: $\mu(E)=M<\infty$. Suppose that $R_{\mu}=[0, M)$. The function $\psi$, which is monotone nonincreasing and continuous on $[0, M)$, can be continuously extended at $M$, and, besides, $\psi(M)=\mathrm{P}\{N(E)=0\}$. Moreover, it can be shown that $N(E)<\infty$ with probability 1 , and that, with $g$ being the generating function of the distribution of $N(E)$,

$$
\psi(a)=g\left(1-\frac{a}{M}\right) \text { for all } 0 \leq a \leq M .
$$

It follows that (3.1) holds for every disjoint measurable set in $\mathcal{E}$. These same results trivially hold when $\tilde{R}_{\mu}=[0, M]$.

In this case, however, the function $\psi$ is not necessarily the Laplace transform of a positive random variable, and so $\mathcal{N}$ need not be a mixed Poisson process. As an illustration, suppose that $\mu(E)=M<\infty$, and choose the total number of points in $E$ according to the shifted geometric distribution

$$
p_{0}(M)=0 \quad \text { and } \quad p_{i}(M)=p q^{i} \quad \text { for } i \geq 1,
$$

where $p$ and $q$ are positive, with $p+q=1$. The generating function of $p(M)$ is $g(z)=$ $p z /(1-q z)$, and $\psi(a)=g(1-a / M)$ is not a Laplace transform because $\psi(M)=0$, and the so-defined multinomial process is not a mixed Poisson process.

\subsection{Sufficient conditions for a Markovian point process to be multinomial}

In Theorems 3.2 and 3.3 below, we explore conditions under which a Markovian point process is multinomial. Loosely speaking, we show that a simple Markovian point process is multinomial.

Theorem 3.2. A simple regular Markovian point process directed by a diffuse measure $\mu$ is a multinomial point process directed by $\mu$.

Proof. Suppose that $A \in \mathcal{B}$ verifies $\mu(A)>0$, and let $B \subseteq A$ be a measurable set. Since $\mu$ is assumed to be diffuse, there exists a nested sequence of partitions $\left\{B_{n, k}\right\}_{k=1, \ldots, 2^{n}}$ of $B$ such that $\mu\left(B_{n, k}\right)=\mu(B) / 2^{n}$. We will prove by induction on $i$ that

$$
\mathrm{P}\{N(B)=j \mid N(A)=i\}=\left(\begin{array}{c}
i \\
j
\end{array}\right)\left(\frac{\mu(B)}{\mu(A)}\right)^{j}\left(1-\frac{\mu(B)}{\mu(A)}\right)^{i-j} \quad \text { for } 0 \leq j \leq i .
$$

For $i=1$, (3.6) is the assertion of Theorem 2.1. Now, assume that (3.6) holds for $i-1$. To simplify the notation, let $P_{i}\{\cdot\}=\mathrm{P}\{\cdot \mid N(A)=i\}$. Then the Markovian character of $\mathcal{N}$ gives

$$
\begin{aligned}
P_{i}\{N(B)=1\} & =\sum_{k=1}^{2^{n}} P_{i}\left\{N\left(B_{n, k}\right)=1\right\} \mathrm{P}\left\{N\left(B-B_{n, k}\right)=0 \mid N\left(A-B_{n, k}\right)=i-1\right\} \\
& =\sum_{k=1}^{2^{n}} P_{i}\left\{N\left(B_{n, k}\right)=1\right\}\left(1-\frac{\mu\left(B-B_{n, k}\right)}{\mu\left(A-B_{n, k}\right)}\right)^{i-1}
\end{aligned}
$$




$$
\begin{aligned}
& =\left(1-\frac{\mu(B)-\mu(B) / 2^{n}}{\mu(A)-\mu(B) / 2^{n}}\right)^{i-1} \sum_{k=1}^{2^{n}} P_{i}\left\{N\left(B_{n, k}\right)=1\right\} \\
& =\left(1-\frac{\mu(B)}{\mu(A)}\right)^{i-1} \lim _{n \rightarrow \infty} \sum_{k=1}^{2^{n}} P_{i}\left\{N\left(B_{n, k}\right)=1\right\} .
\end{aligned}
$$

Similarly, for $j=2, \ldots, i$, we compute the probability $P_{i}\{N(B)=j\}$ as the sum of

(i) the probability that $N(B)=j$ when there exists some $k$ such that $N\left(B_{n, k}\right)>1$, that is, $P_{i}\left\{N(B)=j, N\left(B_{n, k}\right) \geq 2\right.$ for some $\left.k\right\}$, which converges to 0 as $n \rightarrow \infty$ because $\mathcal{N}$ is simple;

(ii) the probability that $N(B)=j$ when there is, at most, one point in each $B_{n, k}$.

The probability in (ii) can be decomposed into contributions from the sets $B_{n, k}$ containing one arbitrarily chosen point among the $j$ points in $B$; to account for this arbitrary choice, we will require a $1 / j$ factor. Furthermore, there must be $j-1$ additional points in $B-B_{n, k}$ and, therefore,

$$
\begin{aligned}
P_{i}\{N(B)=j\}=\varepsilon_{n}+\frac{1}{j} \sum_{k=1}^{2^{n}} & P_{i}\left\{N\left(B_{n, k}\right)=1\right\} \\
\times & \mathrm{P}\left\{N\left(B-B_{n, k}\right)=j-1 \mid N\left(A-B_{n, k}\right)=i-1\right\},
\end{aligned}
$$

where $\varepsilon_{n}$ is the residual term in (i) converging to 0 as $n \rightarrow \infty$. Using the induction hypothesis and letting $n \rightarrow \infty$, we obtain

$$
P_{i}\{N(B)=j\}=\frac{1}{i}\left(\begin{array}{l}
i \\
j
\end{array}\right)\left(\frac{\mu(B)}{\mu(A)}\right)^{j-1}\left(1-\frac{\mu(B)}{\mu(A)}\right)^{i-j} \lim _{n \rightarrow \infty} \sum_{k=1}^{2^{n}} P_{i}\left\{N\left(B_{n, k}\right)=1\right\} .
$$

Let us define $x=\mu(B) / \mu(A)$. Then (3.7) and (3.8) give

$$
P_{i}\{N(B)=j\}=\frac{1}{i}\left(\begin{array}{l}
i \\
j
\end{array}\right)\left(\frac{x}{1-x}\right)^{j-1} P_{i}\{N(B)=1\} \quad \text { for } 0<j \leq i .
$$

To account for the case $j=0$, which is missing in (3.9), observe that, for $D=A-B$, we have

$$
P_{i}\{N(D)=j\}=\frac{1}{i}\left(\begin{array}{l}
i \\
j
\end{array}\right)\left(\frac{1-x}{x}\right)^{j-1} P_{i}\{N(D)=1\},
$$

and, in particular,

$$
\begin{aligned}
P_{i}\{N(B)=0\} & =P_{i}\{N(D)=i\} \\
& =\frac{1}{i}\left(\frac{1-x}{x}\right)^{i-1} P_{i}\{N(B)=i-1\} \\
& =\frac{1}{i} \frac{1-x}{x} P_{i}\{N(B)=1\},
\end{aligned}
$$

thus showing that (3.9) also holds for $j=0$. 
From the fact that $\sum_{j=0}^{i} P_{i}\{N(B)=j\}=1$, we obtain

$$
P_{i}\{N(B)=1\}=i x(1-x)^{i-1},
$$

and, thus,

$$
P_{i}\{N(B)=j\}=\left(\begin{array}{l}
i \\
j
\end{array}\right) x^{j}(1-x)^{i-j} \quad \text { for } 0 \leq j \leq i,
$$

which establishes (3.6). The fact that $\mathcal{N}$ is multinomial easily follows.

Theorem 3.3. A simple homogeneous Markovian point process with respect to a nonpurely atomic measure $\mu$ is multinomial, directed by $\mu$.

Proof. We must prove that the infinitesimal matrix of a simple homogeneous Markovian point process is given by (2.3), and we will prove it by induction on $i$.

Equality (2.2) when $i=1$ was established in Remark 2.1. Suppose now that (2.2) holds for $i-1$, that is, the first $i-1$ rows of $P(x)$ are binomial or, equivalently, the first $i-1$ rows of $Q^{\prime}(0)$ satisfy (2.3).

It is easy to see that the transition matrix of a homogeneous Markovian point process satisfies the Kolmogorov backward differential equations, which in this case are $-x P^{\prime}(x)=Q^{\prime}(0) P(x)$. Then, the backward differential equation for $p_{i, 0}(x)=(1-x)^{q_{i}}$ can be written as

$$
q_{i}(1-x)^{q_{i}-1}=\sum_{j=0}^{i-1} q_{i, j}(1-x)^{j} \quad \text { for } 0 \leq x \leq 1 .
$$

This shows that, for some $0 \leq j<i, q_{i}=q_{i, j}=j+1$, and $q_{i, j^{\prime}}=0$ for $j^{\prime} \neq j$ and $0 \leq j^{\prime}<i$. But, if $1 \leq j<i-1$, the state $i$ communicates with 1 , so that $P_{i, 1}(x)>0$ for all $0<x<1$, and, thus, $P_{i, i-1}(x)>0$ for $0<x<1$, which yields a contradiction. Thus, $P(x)$ does not verify Proposition 2.3(i), except if either $j=0$ or $j=i-1$. If $j=0$ then the $i$ th row of $P(x)$ is $[1-x, 0, \ldots, 0, x]$, and it is easily shown that the process $\mathcal{N}$ is not simple (that is, the $i$ points in $A$ hold together). Therefore, the $i$ th row of $Q^{\prime}(0)$ is necessarily $[0, \ldots, 0, i,-i]$, completing the proof.

As a conclusion, we have shown that a simple homogeneous Markovian point process is (under some additional hypotheses) multinomial, and it is, therefore, characterized by the Laplace transform of a nonnegative random variable when $\mu(E)=\infty$, and by the distribution of $N(E)$ when $\mu(E)<\infty$.

\section{General homogeneous Markovian point processes}

In the previous section we gave conditions under which a Markovian point process is multinomial. Now, we are interested in general Markovian point processes.

\subsection{Characterization of allocation functions}

Before proceeding with our main results in this section, we give some examples of allocation functions that are not of multinomial type (recall (2.2)). In what follows, we suppose that $\mathcal{N}$ is a homogeneous Markovian point process with respect to the boundedly finite, nonpurely atomic measure $\mu$ on $(E, \mathcal{E})$.

Example 4.1. (Binary allocation.) We allocate points in $E$ with weights $1,2,4,8, \ldots$ in positions independently chosen with distribution $\mu(\mathrm{d} x) / \mu(A)$ inside each set $A \in \mathscr{B}$. 
If $N(A)=i$, and $i$ has a binary expression $i=2^{m_{1}}+2^{m_{2}}+\cdots+2^{m_{r}}$, then

$$
P_{i, j}(x)= \begin{cases}(1-x)^{r} & \text { if } j=0, \\ x(1-x)^{r-1} & \text { if } j=2^{m_{1}}, 2^{m_{2}}, \ldots, 2^{m_{r}}, \\ x^{2}(1-x)^{r-2} & \text { if } j=2^{m_{u}}+2^{m_{v}} \text { for some } m_{u}, m_{v}, \\ \vdots & \text { if } j=i .\end{cases}
$$

Thus, the first rows of $Q^{\prime}(0)$ are

\begin{tabular}{r|rrrrrrrr} 
& 0 & 1 & 2 & 3 & 4 & 5 & 6 & 7 \\
\hline 0 & 0 & & & & & & & \\
1 & 1 & -1 & & & & & & \\
2 & 1 & 0 & -1 & & & & & \\
3 & 0 & 1 & 1 & -2 & & & & \\
4 & 1 & 0 & 0 & 0 & -1 & & & \\
5 & 0 & 1 & 0 & 0 & 1 & -2 & & \\
6 & 0 & 0 & 1 & 0 & 1 & 0 & -2 & \\
7 & 0 & 0 & 0 & 1 & 0 & 1 & 1 & -3
\end{tabular}

In general, if $i=2^{m_{1}}+2^{m_{2}}+\cdots+2^{m_{r}}$ then $q_{i}=r$ and $q_{i, i-2^{m_{u}}}=1$, and $q_{i, j}=0$ otherwise.

Example 4.2. (Ternary allocation.) As in the previous example, we allocate points with weights $1,1,3,3,9,9, \ldots$ in positions independently chosen with distribution $\mu(\mathrm{d} x) / \mu(A)$ for each set $A \in \mathscr{B}$.

In this way, if $i=c_{1} 3^{m_{1}}+\cdots+c_{r} 3^{m_{r}}$ then row $i$ has $q_{i, i-3^{m_{u}}}=c_{u}, q_{i, j}=0$ for $j \neq i-3^{m_{u}}$ and, finally, $q_{i}=c_{1}+\cdots+c_{r}$. Therefore, the first rows of $Q^{\prime}(0)$ are

\begin{tabular}{r|rrrrrrrrr} 
& 0 & 1 & 2 & 3 & 4 & 5 & 6 & 7 & \\
\hline 0 & 0 & & & & & & & & \\
1 & 1 & -1 & & & & & & & \\
2 & 0 & 2 & -2 & & & & & & \\
3 & 1 & 0 & 0 & -1 & & & & & \\
4 & 0 & 1 & 0 & 1 & -2 & & & & \\
5 & 0 & 0 & 1 & 0 & 2 & -3 & & & \\
6 & 0 & 0 & 0 & 2 & 0 & 0 & -2 & & \\
7 & 0 & 0 & 0 & 0 & 2 & 0 & 1 & -3 & \\
& $\vdots$ & $\vdots$ & $\vdots$ & & & & & & $\ddots$
\end{tabular}

A careful inspection of the above matrices $Q^{\prime}(0)$ yields the following conclusions.

- There exist some 'batches', that is, points that are allocated together. There exists a batch of size $i$ if $q_{i}=1$.

- When $i$ is not a batch, then $i$ is decomposed as a sum of batches. For instance,

$$
7=1+2+4 \text { or } 7=1+3+3
$$

in the binary and ternary cases, respectively. In both cases, $q_{7}=3$ corresponds to the number of batches in (4.1). Moreover, $q_{i, j}$ equals the number of batches of size $i-j$ in the decomposition of $i$. 
Example 4.3. (Allocation with random choices of the batches.) Consider the infinitesimal matrix

\begin{tabular}{c|ccccccccc} 
& 0 & 1 & 2 & 3 & 4 & 5 & 6 & 7 & \\
\hline 0 & 0 & & & & & & & & \\
1 & 1 & -1 & & & & & & & \\
2 & 1 & 0 & -1 & & & & & & \\
3 & 1 & 0 & 0 & -1 & & & & & \\
4 & 0 & $1-p$ & $2 p$ & $1-p$ & -2 & & & & \\
5 & 1 & 0 & 0 & 0 & 0 & -1 & & & \\
6 & 1 & 0 & 0 & 0 & 0 & 0 & -1 & & \\
7 & 0 & $q$ & $1-q$ & 0 & 0 & $1-q$ & $q$ & -2 & \\
& $\vdots$ & $\vdots$ & & & & & & & $\ddots$
\end{tabular}

for $0<p<1$ and $0<q<1$. The batches correspond to $i=1,2,3,5,6$. When $i=4$, there are two possible decompositions into two batches (indeed, $q_{4}=2$ ):

$$
4=1+3 \text { and } 4=2+2 .
$$

The above matrix corresponds to the case when, with probability $p$, the chosen decomposition is $2+2$ (hence, $q_{4,2}=2 p$ ) and, with probability $1-p$, it is $4=1+3$ (hence, $q_{4,1}=q_{4,3}=1-p$ ). Similarly, for $i=7$, the decomposition can be $7=1+6$ with probability $q$, or $7=2+5$ with probability $1-q$.

4.1.1. General allocation functions. After the preceding examples, our goal is to characterize the form of the allocation functions $P(x)$ and their infinitesimal matrices $Q^{\prime}(0)$.

Lemma 4.1. If $Q^{\prime}(0)$ is the infinitesimal matrix of an allocation function $P(x)$ then

(i) for every $i \geq 1, q_{i}$ is an integer with $q_{i} \leq i$;

(ii) $q_{i, j}=0$ if $q_{j} \neq q_{i}-1$.

Proof. As pointed out in Remark 2.1, $q_{i}>0$ for $i \geq 1$. Also, as in the proof of Theorem 3.3, we can show that

$$
q_{i}(1-x)^{q_{i}-1}=\sum_{j=0}^{i-1} q_{i, j}(1-x)^{q_{j}} \quad \text { for } 0 \leq x \leq 1,
$$

and, since $q_{0}=0$ and $q_{1}=1$, we recurrently find that $q_{i}$ is an integer which is less than or equal to $i$. Furthermore, $q_{i, j}=0$ unless $q_{i}-1=q_{j}$. This completes the proof.

The form of the terms of an allocation function $P(x)$ is now analyzed.

Proposition 4.1. Suppose that $P(x)$ for $0 \leq x \leq 1$ is an allocation function with corresponding infinitesimal matrix $Q^{\prime}(0)$. Then

$$
P_{i, j}(x)=\Pi_{i, j}\left(\begin{array}{c}
q_{i} \\
q_{j}
\end{array}\right) x^{q_{j}}(1-x)^{q_{i}-q_{j}} \quad \text { for } 0 \leq j \leq i \text { and } 0 \leq x \leq 1,
$$

where $\Pi_{i, j}$ is the probability that, starting from $i$, the Markov chain with infinitesimal matrix $Q^{\prime}(0)$ reaches state $j$. In other words, $\Pi_{i, j}$ is the sum of the products $\prod_{l=0}^{r-1} q_{j_{l}, j_{l+1}} / q_{j_{l}}$ for all the decreasing sequences $i=j_{0}>j_{1}>\cdots>j_{r-1}>j_{r}=j$. In particular, $\Pi_{i, 0}=1$. 
Proof. Recall the continuous-time Markov chain interpretation of $P(x)$ and $Q^{\prime}(0)$ given in Remark 2.2. Suppose that the Markov chain with infinitesimal matrix $Q^{\prime}(0)$ is in state $i$ at time $t=0$ (or $x=1$ ). Let us determine the probability that the Markov chain reaches state $j$ at time $t$ after traveling through the states $i=j_{0}, j_{1}, j_{2}, \ldots, j_{r-1}, j_{r}=j$ (in particular, $r=q_{i}-q_{j}$ and $q_{j_{l}}=q_{i}-l$ if this probability is positive). If the jump times are $t_{1}, \ldots, t_{r}$ then the probability of such a path equals

$$
\begin{aligned}
& \int_{0}^{t} \int_{0}^{t_{r}} \cdots \int_{0}^{t_{2}} q_{i} \mathrm{e}^{-q_{i} t_{1}} \frac{q_{i, j_{1}}}{q_{i}}\left(q_{i}-1\right) \mathrm{e}^{-\left(q_{i}-1\right)\left(t_{2}-t_{1}\right)} \frac{q_{j_{1}, j_{2}}}{q_{i}-1} \cdots \\
& \quad \times\left(q_{i}-r+1\right) \mathrm{e}^{-\left(q_{i}-r+1\right)\left(t_{r}-t_{r-1}\right)} \frac{q_{j_{r-1}, j_{r}}}{q_{i}-r+1} \mathrm{e}^{-q_{j}\left(t-t_{r}\right)} \mathrm{d} t_{1} \mathrm{~d} t_{2} \cdots \mathrm{d} t_{r} \\
& =q_{i, j_{1}} q_{j_{1}, j_{2}} \cdots q_{j_{r-1}, j} \mathrm{e}^{-q_{j} t} \int_{0}^{t} \int_{0}^{t_{r}} \cdots \int_{0}^{t_{2}} \mathrm{e}^{-\left(t_{1}+t_{2}+\cdots+t_{r}\right)} \mathrm{d} t_{1} \mathrm{~d} t_{2} \cdots \mathrm{d} t_{r} \\
& =q_{i, j_{1}} q_{j_{1}, j_{2}} \cdots q_{j_{r-1}, j} \mathrm{e}^{-q_{j} t} \frac{1}{r !}\left(1-\mathrm{e}^{-t}\right)^{r} \\
& =\frac{q_{i, j_{1}} q_{j_{1}, j_{2}} \cdots q_{j_{r-1}, j}}{q_{i} ! / q_{j} !}\left(\begin{array}{c}
q_{i} \\
q_{j}
\end{array}\right) \mathrm{e}^{-q_{j} t}\left(1-\mathrm{e}^{-t}\right)^{q_{i}-q_{j}} .
\end{aligned}
$$

Therefore, recalling that $x=\mathrm{e}^{-t}$, the terms of the allocation matrix $P(x)$ are given by

$$
P_{i, j}(x)=\Pi_{i, j}\left(\begin{array}{c}
q_{i} \\
q_{j}
\end{array}\right) x^{q_{j}}(1-x)^{q_{i}-q_{j}},
$$

where $\Pi_{i, j}$ is the sum of the probabilities $q_{i, j_{1}} / q_{i} \cdot q_{j_{1}, j_{2}} / q_{i}-1 \cdots q_{j_{r-1}, j} / q_{j}+1$ for all the sequences of states $i, j_{1}, \ldots, j_{r-1}, j$ leading from $i$ to $j$. In other words, $\Pi_{i, j}$ is the probability of passing through $j$ when starting from $i$, and, in particular, $\Pi_{i, 0}=1$.

We can now give a characterization of the allocation functions and their infinitesimal matrices.

Theorem 4.1. Suppose that $Q^{\prime}(0)=\left(q_{i, j}\right)_{i, j}$ is the infinitesimal matrix of a homogeneous continuous-time Markov chain with state space $\{0,1,2, \ldots\}$ decreasing to the absorbing state 0 . Let the $\{Q(t)\}_{t \geq 0}$ be the corresponding transition matrices, and let $P(x)=Q(-\log x)$ for $0<x \leq 1$ (while $P(0)=P_{0}$ ). The necessary and sufficient conditions for $P(x), 0 \leq x \leq 1$, to be an allocation function are

(i) for every $i \geq 1, q_{i}=-q_{i, i}$ is an integer;

(ii) if $q_{j} \neq q_{i}-1$ then $q_{i, j}=0$;

(iii) if $i \geq 1$ verifies $i=u_{1}+\cdots+u_{r}$, where $u_{1}, \ldots, u_{r}$ are positive integers, then the product

$$
q_{i, i-u_{1}} q_{i-u_{1}, i-u_{1}-u_{2}} \cdots q_{i-u_{1}-u_{2}-\cdots-u_{r-1}, 0}
$$

is invariant under any permutation of $\left(u_{1}, \ldots, u_{r}\right)$.

Proof. The necessity of conditions (i) and (ii) is proved in Lemma 4.1. In order to prove the necessity of (iii), from Proposition 2.3(i) and Proposition 4.1, it follows that, for every $0 \leq x \leq 1$,

$$
\Pi_{i, i-j}\left(\begin{array}{c}
q_{i} \\
q_{i-j}
\end{array}\right)(1-x)^{q_{i-j}} x^{q_{i}-q_{i-j}}=\Pi_{i, j}\left(\begin{array}{c}
q_{i} \\
q_{j}
\end{array}\right) x^{q_{j}}(1-x)^{q_{i}-q_{j}} .
$$


In particular, we obtain

(a) $q_{i}=q_{j}+q_{i-j}$ if $0 \leq j \leq i$ and $\Pi_{i, j}>0$;

(b) $\Pi_{i, i-j}=\Pi_{i, j}$ if $0 \leq j \leq i$;

while Proposition 2.3(ii), together with (b), gives

(c) $\Pi_{i, i-j_{1}} \Pi_{i-j_{1}, i-j_{1}-j_{2}}=\Pi_{i, i-j_{2}} \Pi_{i-j_{2}, i-j_{1}-j_{2}}$ for $0 \leq j_{1}, j_{2} \leq i$.

Finally, observe that $q_{i, i-u}=0$ unless $u \in U=\left\{i \in \mathbb{N} \mid q_{i}=1\right\}$ (see (a)). In this case, it is easily seen that $q_{i, i-u} / q_{i}=\Pi_{i, i-u}$. Therefore, the product in (4.3) is 0 unless $u_{1}, \ldots, u_{r} \in U$. Supposing then that $u_{1}, \ldots, u_{r} \in \mathcal{U}$ in (4.3), and iterating (c), we can show that (iii) holds.

The proof of the sufficiency requires us to show that $P(x)$ verifies conditions (i) and (ii) of Proposition 2.3. Observe first that $q_{0}=0$ and $q_{i}>0$ for $i \geq 1$, since the Markov chain must decrease to the absorbing state 0 . Using the same arguments as in the proof of Proposition 4.1, we can show that the transition probabilities of the continuous-time Markov chain (after the reparametrization $x=\mathrm{e}^{-t}$ ) are given by (4.2). Note also that, according to (ii), the Markov chain with transition matrices $\{Q(t)\}$ evolves through a sequence of states such that the corresponding $q_{i}$ decreases by 1 after each jump; thus, $q_{i} \leq i$.

Suppose that

$$
i=\left(u_{1}+\cdots+u_{r}\right)+\left(u_{r+1}+\cdots+u_{s}\right),
$$

where $u_{r+1}+\cdots+u_{s}=j, s-r=q_{j}$, and $s=q_{i}$. This decomposition represents the sequence of states, traveling from $i$ to 0 through $j$ :

$$
i, i-u_{1}, \ldots, i-u_{1}-\cdots-u_{r}=j, j-u_{r+1}, \ldots, 0 .
$$

If this sequence has a positive probability then, by rearranging the terms in (4.4), it follows from hypothesis (iii) that the decomposition

$$
i=\left(u_{r+1}+\cdots+u_{s}\right)+\left(u_{1}+\cdots+u_{r}\right)
$$

gives a sequence, traveling from $i$ to 0 through $i-j$, which also has positive probability. Hence, $q_{i-j}=r=q_{i}-q_{j}$ whenever we can travel with positive probability from $i$ to $j$, or, in other words,

$$
q_{i}=q_{j}+q_{i-j} \quad \text { if } \Pi_{i, j}>0 .
$$

Thus, $P_{i, j}(1-x)=P_{i, i-j}(x)$ will follow (recall (4.2)) from (4.6) and

$$
\Pi_{i, j}=\Pi_{i, i-j}
$$

However, as pointed out in (4.4) and (4.5), for every sequence leading from $i$ to 0 and passing through $j$, we can construct a sequence leading from $i$ to 0 and passing through $i-j$ which has the same probability. Hence, $\Pi_{i, j} \Pi_{j, 0}=\Pi_{i, i-j} \Pi_{i-j, 0}$, and (4.7) holds, since $\Pi_{j, 0}=$ $\Pi_{i-j, 0}=1$.

By (4.2) and (4.7), to prove the condition in Proposition 2.3(ii), it suffices to show that

$$
\Pi_{i, i-j_{1}} \Pi_{i-j_{1}, j_{2}}=\Pi_{i, i-j_{2}} \Pi_{i-j_{2}, j_{1}} .
$$

The left- and right-hand sides of (4.8) are the probabilities of the sequences leading from $i$ to 0 through $i-j_{1}$ and $j_{2}$, and through $i-j_{2}$ and $j_{1}$, respectively; they are related to the 
decompositions

$$
\begin{aligned}
& i=\left(u_{1}+\cdots+u_{r}\right)+\left(u_{r+1}+\cdots+u_{s}\right)+\left(u_{s+1}+\cdots+u_{t}\right), \\
& i=\left(u_{s+1}+\cdots+u_{t}\right)+\left(u_{r+1}+\cdots+u_{s}\right)+\left(u_{1}+\cdots+u_{r}\right),
\end{aligned}
$$

where $u_{1}, \ldots, u_{t} \in U, u_{1}+\cdots+u_{r}=j_{1}$, and $u_{s+1}+\cdots+u_{t}=j_{2}$. Since both have the same probability, as a consequence of hypothesis (iii), we obtain

$$
\Pi_{i, i-j_{1}} \Pi_{i-j_{1}, j_{2}} \Pi_{j_{2}, 0}=\Pi_{i, i-j_{2}} \Pi_{i-j_{2}, j_{1}} \Pi_{j_{1}, 0},
$$

and (4.8) holds.

Remark 4.1. Another consequence of the proof of Theorem 4.1 is that the product in (4.3) is 0 unless the $u_{l}$ are in $U=\left\{i \in \mathbb{N} \mid q_{i}=1\right\}$. Therefore, it suffices to prove the permutationinvariant property only when $u_{1}, \ldots, u_{r} \in \mathcal{U}$.

It is worth noting that Theorem 4.1 does not assert that a homogeneous Markovian process can be constructed from any $Q$-matrix satisfying the conditions (i)-(iii). Indeed, it remains to choose a family of marginal distributions $\{p(a)\}$ compatible with $P(x)$; see Proposition 2.3.

Example 4.4. Consider the infinitesimal matrix

$$
\left[\begin{array}{cccccccccc}
0 & & & & & & & & \\
1 & -1 & & & & & & & \\
1 & 0 & -1 & & & & & & \\
1 & 0 & 0 & -1 & & & & & \\
0 & 1-p & 2 p & 1-p & -2 & & & & \\
0 & 0 & 1 & 1 & 0 & -2 & & & \\
0 & 0 & 0 & 2 & 0 & 0 & -2 & & \\
0 & 0 & 0 & 0 & p_{1} & p_{2} & p_{3} & -3 & \\
\vdots & & & & & & & & \ddots
\end{array}\right]
$$

where $0<p<1$ and $p_{1}+p_{2}+p_{3}=3$. We have $\{1,2,3\} \subseteq \mathcal{U}$.

According to this matrix, $i=4$ can be decomposed either as $4=2+2$ (with probability $p$ ) or $4=3+1$ (with probability $1-p$ ). For $i=5$ and $i=6$, the only possible decompositions are $5=3+2$ and $6=3+3$. For $i=7$, there are two possibilities: $7=1+3+3$ and $7=2+2+3$. However, in order to fulfill Theorem 4.1(iii), the corresponding probabilities must be related in some way to $p$. More precisely, the equality of the products in (4.3) corresponding to the decompositions

$$
7= \begin{cases}1+3+3 & \text { with probability } p_{3} \cdot 2 \cdot 1 \\ 3+1+3 & \text { with probability } p_{1} \cdot(1-p) \cdot 1 \\ 3+3+1 & \text { with probability } p_{1} \cdot(1-p) \cdot 1\end{cases}
$$

gives $2 p_{3}=p_{1}(1-p)$. Similarly, from the decompositions

$$
7= \begin{cases}2+2+3 & \text { with probability } p_{2} \cdot 1 \cdot 1 \\ 2+3+2 & \text { with probability } p_{2} \cdot 1 \cdot 1 \\ 3+2+2 & \text { with probability } p_{1} \cdot 2 p \cdot 1\end{cases}
$$


we obtain $p_{2}=2 p \cdot p_{1}$. Recalling that $p_{1}+p_{2}+p_{3}=3$, it follows that

$$
p_{1}=\frac{2}{1+p}, \quad p_{2}=\frac{4 p}{1+p}, \quad \text { and } \quad p_{3}=\frac{1-p}{1+p}
$$

is the unique row, for $i=7$, compatible with the preceding rows.

\subsection{Marked multinomial processes}

In this section we explore the relationship existing between homogeneous Markovian processes and marked multinomial processes. First of all, we show how to construct a Markovian point process on a bounded measurable set.

Theorem 4.2. Let $Q^{\prime}(0)$ be the infinitesimal matrix associated to a homogeneous Markovian point process $\mathcal{N}$ with respect to a boundedly finite, nonpurely atomic measure $\mu$. Given $A \in \mathscr{B}$, let us define the homogeneous Markovian point process $\mathcal{N}^{\prime}$ on A as follows.

- Suppose that $N(A)=i$ for some $i \geq 1$.

- Select $q_{i}$ random points in $A$, denoted by $X_{1}, \ldots X_{q_{i}}$, independent and identically distributed with distribution given by $\mu(\mathrm{d} x) / \mu(A)$.

- Assign to $X_{1}$ a weight $k_{1}$ chosen at random with distribution $\left\{q_{i, i-k} / q_{i}\right\}_{k \geq 1}$.

- Assign to $X_{2}$ a weight $k_{2}$ chosen with distribution $\left\{q_{i-k_{1}, i-k_{1}-k} / q_{i-k_{1}}\right\}_{k \geq 1}$ conditional on $k_{1}$ (recall that $q_{i-k_{1}}=q_{i}-1$ ).

- Assign to $X_{q_{i}-1}$ a weight $k_{q_{i}-1}$ chosen with distribution

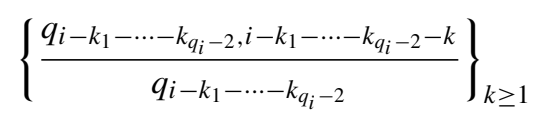

conditional on $k_{1}, \ldots, k_{q_{i}-2}$ (recall that $\left.q_{i-k_{1}-\cdots-k_{q_{i}-2}}=2\right)$.

- Assign to $X_{q_{i}}$ a weight $i-k_{1}-\cdots-k_{q_{i}-1}$.

Finally, define

$$
N^{\prime}(B)=\sum_{r=1}^{q_{i}} k_{r} \delta_{X_{r}}(B) \quad \text { for } B \subseteq A \text { in } \mathcal{B} .
$$

Then, the point processes $\{N(B)\}_{B \subseteq A}$, conditional on $N(A)=i$, and $\mathcal{N}^{\prime}$ have the same distribution.

Proof. According to Theorem 4.1(iii), the joint distribution of the weights assigned to the points $X_{1}, \ldots, X_{q_{i}}$ is invariant under any permutation of the indices.

Now $\left\{N^{\prime}(B)\right\}_{B \in \mathcal{E}_{A}}$ is a Markovian point process. In fact, for any $C \subseteq A$ and, conditional on $N^{\prime}(C)=j$, there are $q_{j}$ points inside $C$ (say, $X_{1}, \ldots, X_{q_{j}}$, since their weights do not depend on their order) and $q_{i-j}=q_{i}-q_{j}$ points in $A-C$ (say, $X_{q_{j}+1}, \ldots, X_{q_{i}-q_{j}}$ ); they all have independent positions and, moreover, the weights $k_{q_{j}+1}, \ldots, k_{q_{i}}$ are independent of $k_{1}, \ldots, k_{q_{j}}$, once $k_{1}+\cdots+k_{q_{j}}=j$ is fixed. Therefore, $N^{\prime}\left(B_{1}\right), \ldots, N^{\prime}\left(B_{r}\right)$ and $N^{\prime}\left(B_{1}^{\prime}\right), \ldots, N^{\prime}\left(B_{s}^{\prime}\right)$ are independent if $B_{1}, \ldots, B_{r} \subseteq C$ and $B_{1}^{\prime}, \ldots, B_{s}^{\prime} \subseteq A-C$.

Furthermore, $\mathcal{N}^{\prime}$ is homogeneous with respect to $\mu$ because, if $B \subseteq C$ is such that $\mu(B)=$ $x \mu(C)$, it is

$$
\mathrm{P}\left\{N^{\prime}(B)=h \mid N^{\prime}(C)=j\right\}=\left(\begin{array}{c}
q_{j} \\
q_{h}
\end{array}\right) x^{q_{h}}(1-x)^{q_{j}-q_{h}} \Pi_{j, h},
$$


since the first term is the probability that exactly $q_{h}$ of the $q_{j}$ points lie in $B$, and $\Pi_{j, h}$ is the probability that the weights of these $q_{h}$ points sum up a total weight $h$. Hence, inside $A, \mathcal{N}^{\prime}$ is a homogeneous Markovian point process with the same allocation function as $\mathcal{N}$.

Let $\mathcal{N}$ be a homogeneous Markovian point process with respect to a boundedly finite, nonpurely atomic measure $\mu$. We define the point process $\tilde{N}$ as

$$
\tilde{N}=q_{\mathcal{N}} .
$$

More explicitly, given a bounded set $A \in \mathcal{B}$, we define $\tilde{N}(A)=q_{N(A)}$, which gives the number of batches in $A$. Observe that, conditional on $N(A)=i$, the point processes

$$
\tilde{N}^{\prime}(B)=\sum_{r=1}^{q_{i}} \delta_{X_{r}}(B) \quad \text { for } B \subseteq A
$$

(using the notation in Theorem 4.2) and $\tilde{N}$ have the same distribution.

Remark 4.2. If the measure $\mu$ is diffuse then $\tilde{N}$ is the support process of $\mathcal{N}$; see [3, Section 7.1]. If $\mu$ is not diffuse, however, then $\tilde{N}$ might not be a simple point process, thus being different from the support process of $\mathcal{N}$.

In our next result, we prove that $\tilde{N}$ in (4.9) is a multinomial point process.

Theorem 4.3. Let $\mathcal{N}$ be a homogeneous Markovian point process with respect to a boundedly finite, nonpurely atomic measure $\mu$. Then $\tilde{N}$ is a multinomial point process with respect to $\mu$.

Proof. Given disjoint bounded measurable sets $A_{1}, \ldots, A_{k}$ and nonnegative integers $r_{1}, \ldots$, $r_{k}$, let $A=\bigcup_{i=1}^{k} A_{i}, r=r_{1}+\cdots+r_{k}$, and define the set of indices

$$
I_{j}\left(r_{1}, \ldots, r_{k}\right)=\left\{\left(i_{1}, \ldots, i_{k}\right) \mid q_{i_{1}}=r_{1}, \ldots, q_{i_{k}}=r_{k}, i_{1}+\cdots+i_{k}=j\right\} .
$$

Then we have

$$
\mathrm{P}\left\{\tilde{N}\left(A_{1}\right)=r_{1}, \ldots, \tilde{N}\left(A_{k}\right)=r_{k}\right\}=\sum_{\left\{j \mid q_{j}=r\right\}} \sum_{I_{j}\left(r_{1}, \ldots, r_{k}\right)} \mathrm{P}\left\{N\left(A_{1}\right)=i_{1}, \ldots, N\left(A_{k}\right)=i_{k}\right\} .
$$

However, as a consequence of Proposition 4.1, the terms of this sum are given by

$$
\begin{aligned}
& \mathrm{P}\left\{N(A)=i_{1}+\cdots+i_{k}\right\}\left(\begin{array}{c}
r \\
r_{1} \cdots r_{k}
\end{array}\right)\left(\frac{\mu\left(A_{1}\right)}{\mu(A)}\right)^{r_{1}} \cdots\left(\frac{\mu\left(A_{k}\right)}{\mu(A)}\right)^{r_{k}} \\
& \times \Pi_{i_{1}+\cdots+i_{k}, i_{2}+\cdots+i_{k}} \Pi_{i_{2}+\cdots+i_{k}, i_{3}+\cdots+i_{k}} \cdots \Pi_{i_{k}, 0} .
\end{aligned}
$$

Summing up in $\left(i_{1}, \ldots, i_{k}\right) \in I_{j}\left(r_{1}, \ldots, r_{k}\right)$, the above expression yields

$$
\mathrm{P}\{N(A)=j\}\left(\begin{array}{c}
r \\
r_{1} \cdots r_{k}
\end{array}\right)\left(\frac{\mu\left(A_{1}\right)}{\mu(A)}\right)^{r_{1}} \cdots\left(\frac{\mu\left(A_{k}\right)}{\mu(A)}\right)^{r_{k}} .
$$

Therefore, the probability $\mathrm{P}\left\{\tilde{N}\left(A_{1}\right)=r_{1}, \ldots, \tilde{N}\left(A_{k}\right)=r_{k}\right\}$ equals

$$
\mathrm{P}\{\tilde{N}(A)=r\}\left(\begin{array}{c}
r \\
r_{1} \cdots r_{k}
\end{array}\right)\left(\frac{\mu\left(A_{1}\right)}{\mu(A)}\right)^{r_{1}} \cdots\left(\frac{\mu\left(A_{k}\right)}{\mu(A)}\right)^{r_{k}} .
$$

From this, it is easily proved that $\tilde{N}$ is multinomial with respect to $\mu$. 
Our next result shows that, under suitable assumptions, a Markovian point process is a marked multinomial process.

Theorem 4.4. Suppose that $\mathcal{N}$ is a homogeneous Markovian point process with respect to a boundedly finite, nonpurely atomic measure $\mu$. Suppose further that either the corresponding infinitesimal matrix $Q^{\prime}(0)$ is integer valued or $\mu$ is diffuse. Then $\mathcal{N}$ is a marked multinomial point process.

Proof. Suppose first that $\mu$ is a diffuse measure. Then, since $\tilde{N}$ is a multinomial point process, it is simple. Thus, P-almost surely, the sample path $\tilde{N}_{\omega}$ is characterized by a sequence $\left\{x_{n}\right\}$ of points in $E$ (with no accumulation points); to each of them $\mathcal{N}_{\omega}$ associates a weight $w_{n}=\mathcal{N}_{\omega}\left(x_{n}\right)$. Now define $\hat{N}_{\omega}$ to be the boundedly finite, integer-valued measure on $E \times \mathbb{N}$ given by

$$
\hat{N}_{\omega}\left\{\left(x_{n}, w_{n}\right)\right\}=1 .
$$

It follows from [3, Definition 7.1.XII] that $\hat{N}$ is a marked point process, whose first component $\hat{N}(A \times \mathbb{N})=\tilde{N}(A)$ is a multinomial point process. Since there is an obvious bijective correspondence between the paths of $\hat{N}$ and $\mathcal{N}, \mathcal{N}$ itself may be considered as a marked multinomial point process.

If $\mu$ is not diffuse, $\tilde{N}$ is not simple; so that, for each $\omega \in \Omega$, there may exist points in the sequence $\left\{x_{n}\right\}$ with multiplicity $\tilde{N}_{\omega}\left(x_{n}\right)=k$. However, if the infinitesimal matrix $Q^{\prime}(0)$ is integer valued, the value $N_{\omega}\left(x_{n}\right)$ can be uniquely decomposed as a sum of $k$ terms: $N_{\omega}\left(x_{n}\right)=w_{1}+\cdots+w_{k}$ (see, e.g. the binary and ternary allocation functions in Section 4.1). Thus, we can define

$$
\hat{N}_{\omega}\left\{\left(x_{n}, w_{j}\right)\right\}=1 \quad \text { for } 1 \leq j \leq k,
$$

and the proof proceeds as in the previous case.

Regarding the restrictions of the above theorem, suppose that $\mu$ is not diffuse and that the infinitesimal matrix $Q^{\prime}(0)$ is not integer valued. As an illustration, consider the infinitesimal matrix in Example 4.3. Given a sample $N_{\omega}$, suppose that we have $N_{\omega}(A)=4$ for some $A \in \mathscr{B}$. Then

$$
\tilde{N}_{\omega}(A)=q_{N_{\omega}(A)}=q_{4}=2
$$

(that is, there are two batches $x_{1}$ and $x_{2}$ in $A$ with corresponding weights $w_{1}$ and $w_{2}$, such that $\left.w_{1}+w_{2}=4\right)$. But, $\mu$ not being diffuse, the two batches may coincide: $x=x_{1}=x_{2}$. In this case, $N_{\omega}\{x\}=4$, although the weights $w_{1}$ and $w_{2}$ are unidentifiable; indeed, they might either be $w_{1}=1$ and $w_{2}=3$, or $w_{1}=w_{2}=2$. In other words, in the case that $\mu$ is not diffuse and $Q^{\prime}(0)$ is not integer valued, from the sole observation of $\mathcal{N}$ we cannot determine $\hat{N}$ as defined in the proof of Theorem 4.4 .

\subsection{Marginal distributions of a homogeneous Markovian point process}

Let $\mathcal{N}$ be a homogeneous Markovian point process with respect to the boundedly finite, nonpurely atomic measure $\mu$. The issue of the marginal distributions of $\mathcal{N}$ is of no interest when $\mu(E)=M<\infty$ because, in this case, we can choose an arbitrary probability vector $p(M)$, and then define

$$
p(a)=p(M) P\left(\frac{a}{M}\right) \text { for } a \in R_{\mu} .
$$

Therefore, our subsequent results are only relevant in the case $\mu(E)=\infty$, although they are true whether $\mu(E)$ is finite or not. 
Let $Q^{\prime}(0)$ be the infinitesimal matrix associated to the allocation function of $\mathcal{N}$. We know from Proposition 2.3(v) that, given $a>0$ and $0 \leq x \leq 1$,

$$
p(a x)=p(a) P(x) \quad \text { if } a x \prec a .
$$

Therefore, $p(a)$ satisfies the backward Kolmogorov differential equations, which in this case take the form $-a p^{\prime}(a)=p(a) Q^{\prime}(0)$, i.e.

$$
-a p_{j}^{\prime}(a)=-q_{j} p_{j}(a)+\sum_{\left\{i \mid q_{i}=q_{j}+1\right\}} p_{i}(a) q_{i, j} \quad \text { for } j \geq 0 .
$$

It is worth noting that $p(a)$ is defined when $a \in R_{\mu}$, and, thus, the derivative of $p(a)$ with respect to $a$ might not make sense. However, we may take (4.10) as a definition (which will coincide, when $a x \prec a$, with the distribution of $\mathcal{N}$ on a set with $\mu$-measure $a x$ ), and then the derivative in (4.11) exists for $a \in(0, \mu(E))$.

On the other hand, for the multinomial point process $\tilde{N}$ defined in (4.9), let us denote its marginal distribution by $\tilde{p}_{q}(a)=\mathrm{P}\{\tilde{N}(A)=q\}$ when $\mu(A)=a$. They verify the corresponding backward Kolmogorov differential equations:

$$
-a{\tilde{p_{q}}}^{\prime}(a)=-q \tilde{p}_{q}(a)+(q+1) \tilde{p}_{q+1}(a) \text { for } q \geq 0 \text { and } a \in(0, \mu(E)) .
$$

Let us now define the conditional probabilities

$$
w_{i}(a)=\mathrm{P}\left\{N(A)=i \mid \tilde{N}(A)=q_{i}\right\}=\frac{p_{i}(a)}{\tilde{p}_{q_{i}}(a)} \quad \text { for } i \geq 0,
$$

which can be arranged in a nonnegative row vector $\boldsymbol{w}(a)=\left(w_{i}(a)\right)_{i \geq 0}$ such that

$$
\sum_{\left\{i \mid q_{i}=q\right\}} w_{i}(a)=1 \quad \text { for all } q \geq 0 \text { and } a \in(0, \mu(E)) .
$$

From (4.11), (4.12), and (4.13), for all $j \geq 0$ and $a \in(0, \mu(E))$, we obtain

$$
w_{j}^{\prime}(a)=\frac{\tilde{p}_{q_{j}+1}(a)\left(q_{j}+1\right)}{-a \tilde{p}_{q_{j}}(a)}\left(\sum_{\left\{i \mid q_{i}=q_{j}+1\right\}} w_{i}(a) t_{i, j}-w_{j}(a)\right),
$$

where the $t_{i, j}$ are the terms of the jump matrix $T$ associated to $Q$ (that is, $t_{0,0}=1, t_{i, j}=q_{i, j} / q_{i}$ if $0 \leq j<i$, and $t_{i, j}=0$ otherwise).

The differential equation (4.14) suggests that we can obtain constant solutions when the expression within the parentheses vanishes. This is made clear in our next result.

Theorem 4.5. Let $\tilde{N}$ be a multinomial process with respect to a measure $\mu$ with marginal distributions $\tilde{p}(a)$ for $a \in R_{\mu}$. Suppose that $Q^{\prime}(0)$ is the infinitesimal matrix of an allocation function $P(x)$ for $0 \leq x \leq 1$, and suppose that the row vector $\boldsymbol{w}$ is a solution of the linear system of equations

$$
\boldsymbol{w}=\boldsymbol{w} T
$$

with $w_{i} \geq 0$ and $\sum_{\left\{i \mid q_{i}=q\right\}} w_{i}=1$ for all $q \geq 0$, where $T$ is the jump matrix of $Q^{\prime}(0)$.

Then $p_{i}(a)=w_{i} \tilde{p}_{q_{i}}(a)$ for $i \geq 0$ and $a \in R_{\mu}$ are the marginal probabilities of $a$ homogeneous Markovian process with respect to $\mu$, with allocation function given by $Q^{\prime}(0)$, and with the same avoidance function as $\tilde{N}$. 
Proof. Note first that

$$
\Pi_{i, j}=\left(T^{q_{i}-q_{j}}\right)_{i, j}, \quad \text { and, thus, } \quad \Pi=\sum_{n=0}^{\infty} T^{n} .
$$

Therefore, if $\boldsymbol{w}=\boldsymbol{w} T$ then $\boldsymbol{w}=\boldsymbol{w} T^{n}$ for all $n$, and $\boldsymbol{w}$ verifies

$$
\sum_{\left\{i \mid q_{i}=q\right\}} w_{i} \Pi_{i, j}=w_{j} \quad \text { for all } j \text { and } q \geq q_{j} .
$$

Now, fix $a \in R_{\mu}$ and $a x \prec x$ for $0 \leq x \leq 1$. The $j$ th term of $p(a) P(x)$ is

$$
\begin{aligned}
\sum_{i=j}^{\infty} p_{i}(a) \Pi_{i, j}\left(\begin{array}{c}
q_{i} \\
q_{j}
\end{array}\right) x^{q_{j}}(1-x)^{q_{i}-q_{j}} & =\sum_{q=q_{j}}^{\infty} \tilde{p}_{q}(a)\left(\begin{array}{c}
q \\
q_{j}
\end{array}\right) x^{q_{j}}(1-x)^{q-q_{j}} \sum_{\left\{i \mid q_{i}=q\right\}} w_{i} \Pi_{i, j} \\
& =w_{j} \tilde{p}_{q_{j}}(a x) \\
& =p_{j}(a x) ;
\end{aligned}
$$

hence, $p(a x)=p(a) P(a x)$ and, by Proposition 2.3, the stated result holds.

In our next result, we prove that when the infinitesimal matrix $Q^{\prime}(0)$ has a finite number of 'batches', then solutions to $\boldsymbol{w}=\boldsymbol{w} T$ exist.

Proposition 4.2. Let $Q^{\prime}(0)$ be the infinitesimal matrix of an allocation function, and let $T$ be the corresponding jump matrix. Suppose that $U=\left\{i \mid q_{i}=1\right\}$ is a finite set. Then there exists a solution to

$$
\boldsymbol{w}=\boldsymbol{w} T, \quad \boldsymbol{w} \geq \mathbf{0}, \quad \text { and } \quad \sum_{\left\{i \mid q_{i}=q\right\}} w_{i}=1 \quad \text { for all } q \geq 0 .
$$

Proof. Let us rearrange the integers $\{0,1,2,3, \ldots\}$ as

$$
0,\left\{i \mid q_{i}=1\right\},\left\{i \mid q_{i}=2\right\}, \ldots,
$$

where, as a consequence of our hypotheses, the above sets are all finite. In this way, the jump matrix $T$ adopts a block lower-triangular form:

$$
T=\left[\begin{array}{ccccc}
1 & 0 & 0 & 0 & \cdots \\
T_{1,0} & 0 & 0 & 0 & \cdots \\
0 & T_{2,1} & 0 & 0 & \cdots \\
0 & 0 & T_{3,2} & 0 & \cdots \\
\vdots & & & & \ddots
\end{array}\right]
$$

Now fix an arbitrary sequence $\left\{\boldsymbol{v}^{(q)}\right\}_{q \geq 1}$ of probability vectors on $\left\{i \mid q_{i}=q\right\}$. Given $j \geq 0$, define $\boldsymbol{w}_{j}^{(q)}=\boldsymbol{v}^{(q)} T^{q-j}$ for $q \geq j$, which is a probability vector on $\left\{i \mid q_{i}=j\right\}$. Now $\left\{\boldsymbol{w}_{1}^{(q)}\right\}_{q \geq 1}$ is a sequence of probability vectors on the finite set $\left\{i \mid q_{i}=1\right\}$, and, therefore, it has a subsequence $\left\{\boldsymbol{w}_{1}^{\left(q^{\prime}\right)}\right\}$ converging to some probability vector $\boldsymbol{w}_{1}^{*}$. Similarly, $\left\{\boldsymbol{w}_{2}^{\left(q^{\prime}\right)}\right\}$ has a subsequence $\left\{\boldsymbol{w}_{2}^{\left(q^{\prime \prime}\right)}\right\}$ converging to some probability vector $\boldsymbol{w}_{2}^{*}$ on $\left\{i \mid q_{i}=2\right\}$. Using a diagonal argument, we can show that there exists a subsequence $\left\{q^{*}\right\}$ such that $\boldsymbol{w}_{j}^{\left(q^{*}\right)}$ converges to $\boldsymbol{w}_{j}^{*}$, which is a probability vector on $\left\{i \mid q_{i}=j\right\}$ for all $j \geq 1$. It also follows that

$$
\boldsymbol{w}_{j}^{*}=\boldsymbol{w}_{j+1}^{*} T_{j+1, j},
$$

and, therefore, $\boldsymbol{w}^{*}=\left(1, \boldsymbol{w}_{1}^{*}, \boldsymbol{w}_{2}^{*}, \ldots\right)$ verifies the stated result. 
Let us now analyze the existence of nonconstant solutions to (4.14). For $j \geq 0$ and $a \in$ $(0, \mu(E))$, define

$$
v_{j}(a)=p_{j}(a) \frac{q_{j} !}{a^{q_{j}}} .
$$

Note that, for $a=0$, the definition is made by continuity. Using (4.11), it is easily shown that

$$
v_{j}^{\prime}(a)=-\sum_{\left\{i \mid q_{i}=q_{j}+1\right\}} v_{i}(a) t_{i, j} \quad \text { for } j \geq 0 \text { and } a \in(0, \mu(E)) .
$$

In vector notation, letting $\boldsymbol{v}(a)=\left\{v_{j}(a)\right\}_{j \geq 0}$, it follows that $\boldsymbol{v}(a)$ is a solution of the linear system of differential equations $\boldsymbol{v}^{\prime}(a)=-\boldsymbol{v}(a) T$ for $a \in(0, \mu(E))$. This fact allows us to state the following result.

Theorem 4.6. Suppose that $T$ is the jump matrix of the infinitesimal matrix $Q^{\prime}(0)$ of an allocation function. Suppose further that there exists some vector $v$ such that $v(a)=v \mathrm{e}^{-a T}$ verifies $v_{j}(a) \geq 0$ for all $a \in(0, \mu(E))$ and $j \geq 0$, and

$$
\sum_{j=0}^{\infty} v_{j}(a) \frac{a^{q_{j}}}{q_{j} !}=1 \quad \text { for all } a \in(0, \mu(E))
$$

Then

$$
p_{j}(a)=v_{j}(a) \frac{a^{q_{j}}}{q_{j} !} \quad \text { for } j \geq 0 \text { and } a \in(0, \mu(E))
$$

is the marginal probability function of a homogeneous Markovian point process with jump matrix $T$.

Proof. The proof is made by direct substitution of (4.15) in

$$
p_{j}(a x)=\sum_{i=j}^{\infty} p_{i}(a) \Pi_{i, j}\left(\begin{array}{c}
q_{i} \\
q_{j}
\end{array}\right) x^{q_{j}}(1-x)^{q_{i}-q_{j}},
$$

and using the fact that $\boldsymbol{v}(a x)=\boldsymbol{v}(a) \mathrm{e}^{a(1-x) T}$ for $a \geq 0$ and $0 \leq x \leq 1$.

Observe that if $\mu(E)=M<\infty$ then $p(M)$ determines $v(M)$, and $v=v(M) \mathrm{e}^{M T}$ satisfies the hypotheses of Theorem 4.6. On the other hand, when $\mu(E)=\infty$, it will be interesting to know whether the existence of such a vector $v$ can be granted, or the only available marginal distributions are as in Theorem 4.5.

\section{Conclusions}

In this paper we have presented a self-contained survey of Markovian point processes, as defined in Section 2. With respect to the homogeneous Markovian point processes, we have given an exhaustive description of their allocation functions by means of the infinitesimal matrix $Q^{\prime}(0)$. Furthermore, they have been characterized as marked multinomial point processes with marks attributed as indicated by the matrix $Q^{\prime}(0)$.

Our analysis of the marginal distributions of general homogeneous Markovian point processes is not that thorough, and we have only given sufficient conditions (in Theorems 4.5 and 4.6) yielding the aforementioned marginal distributions. Further research on this issue is currently in progress. 
Summarizing, under the hypotheses of Theorem 4.5, a general homogeneous Markovian point process $\mathcal{N}$ with respect to a fixed boundedly finite measure $\mu$, with $\mu(E)=\infty$, may be constructed from the following basic elements:

(a) an avoidance function $\psi$, which is the Laplace transform of a nonnegative random variable, yielding the marginal distributions $\tilde{p}_{q}(a)$ of the underlying multinomial process $\tilde{N}$;

(b) a solution of $\boldsymbol{w}=\boldsymbol{w} T$ (with $w_{i} \geq 0$ and $\sum_{\left\{i \mid q_{i}=q\right\}} w_{i}=1$ ) which, together with $\tilde{p}_{q}(a)$, determines the marginal distributions of $\mathcal{N}: p_{i}(a)=w_{i} \tilde{p}_{q_{i}}(a)$ (or, also, a vector $\boldsymbol{v}$ satisfying Theorem 4.6, which allows us to determine the marginal distributions $p_{i}(a)$ by means of (4.15));

(c) an infinitesimal matrix $Q^{\prime}(0)$, as in Theorem 4.1, which, conditional on $N(A)$, allows us to assign weights to the points in each set $A \in \mathscr{B}$ by using the procedure described in Theorem 4.2.

Let us now mention some interesting open issues. It would be interesting to know whether all the results in this paper could be extended to Markovian processes defined on an abstract measurable space (instead of complete and separable metric spaces). In this sense, reference [7], which deals with Poisson processes defined on general measurable spaces, may be enlightening.

Another challenging issue is the statistical inference for Markovian point processes [6]. It is easily shown (and, moreover, it is quite intuitive) that, given the observation of $\mathcal{N}$ on $A \in \mathscr{B}$, $N(A)$ is a sufficient statistic. The convergence (3.5) shows that consistent estimators cannot be derived from $N(A)$ as $A \uparrow E$. This is a very important departure point from the results on Poisson processes, for which the limit in (3.5) is a constant. This shows that the statistical inference issue for Markovian point process may be an awkward problem, and, thus, worth taking care of.

\section{Acknowledgement}

This paper is a posthumous tribute to Professor Miguel Martín Díaz, who died unexpectedly on June 5th 2006, just before his retirement. His advice and suggestions were of great help to us during the preparation of this manuscript. His death is an irremediable loss for all his disciples, colleagues, and friends. Rest in peace.

\section{References}

[1] Chow, Y. S. And Teicher, H. (1988). Probability Theory, 2nd edn. Springer, New York.

[2] Chung, K. L. (1967). Markov Chains with Stationary Transition Probabilities. Springer, New York.

[3] Daley, D. J. And Vere-Jones, D. (1988). An Introduction to the Theory of Point Processes. Springer, New York.

[4] Diggle, P. J. (2003). Statistical Analysis of Spatial Point Patterns, 2nd edn. Edward Arnold, London.

[5] Kallenberg, O. (1983). Random Measures, 3rd edn. Academic Press, London.

[6] Karr, A. F. (1991). Point Processes and Their Statistical Inference, 2nd edn. Marcel Dekker, New York.

[7] Kingman, J. F. C. (1993). Poisson Processes. Oxford Univerity Press, New York.

[8] Moyal, J. E. (1962). The general theory of stochastic population processes. Acta Math. 108, 1-31.

[9] Van Lieshout, M. N. M. (2000). Markov Point Processes and Their Applications. Imperial College Press, London. 